\title{
Green Tea Consumption and Risk of Breast Cancer and Recurrence-A Systematic Review and Meta-Analysis of Observational Studies
}

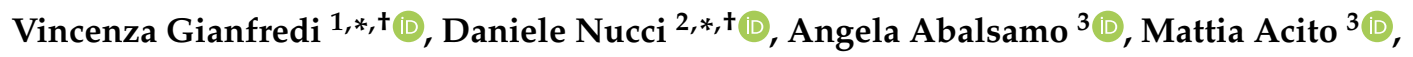

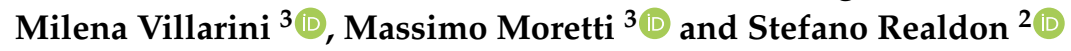 \\ 1 Post-Graduate School of Hygiene and Preventive Medicine, Department of Experimental Medicine, \\ University of Perugia, P.le L. Severi 1, 06132 Perugia, Italy \\ 2 Digestive Endoscopy Unit, Veneto Institute of Oncology IOV-IRCCS, Via Gattamelata 64, 35128 Padua, Italy; \\ stefano.realdon@iov.veneto.it \\ 3 Department of Pharmaceutical Science, University of Perugia, Via del Giochetto 2, 06123 Perugia, Italy; \\ angela.abalsamo@yahoo.it (A.A); mattia.acito@studenti.unipg.it (M.A.); milena.villarini@unipg.it (M.V.); \\ massimo.moretti@unipg.it (M.M.) \\ * Correspondence: vincenza.gianfredi@studenti.unipg.it (V.G.); daniele.nucci@iov.veneto.it (D.N.); \\ Tel.: +39-075-585-7488 (V.G.); +39-049-8221-1719 (D.N.); Fax: +39-075-585-7422 (V.G.) \\ + These two authors equally contributed to the manuscript.
}

Received: 10 November 2018; Accepted: 28 November 2018; Published: 3 December 2018

\begin{abstract}
Breast cancer (BC) is the most common cancer in women and several factors are involved in its onset. Green tea (GT) has been shown to have potential beneficial effects on different types of cancer. The aim of this review was to evaluate the association between GT regular consumption and risk of $\mathrm{BC}$ in women. The risk of $\mathrm{BC}$ recurrence and risk of $\mathrm{BC}$ in relation to menopausal status were also evaluated. A literature search of PubMed, Scopus, and Web of Science was conducted. Preferred Reporting Items for Systematic Reviews and Meta-Analyses (PRISMA) guidelines were followed to perform the systematic review and meta-analysis. Full texts were downloaded for 40 studies; however, only 13 records were included in the meta-analysis. Eight were cohort studies and five were case-control studies. The pooled sample consisted of 163,810 people. An inverse statistically significant relationship between GT and BC risk, with an Odds Ratio (OR) $=0.85((95 \%$ $\mathrm{CI}=0.80-0.92), p=0.000)$ ), was found. Egger's linear regression test did not show a potential publication bias (intercept $0.33, t=0.40, p=0.695$ ), which was also confirmed by the symmetry of the funnel plot. Moreover, no high statistical heterogeneity $\left(\mathrm{Chi}^{2}=31.55, d f=13, I^{2}=58.79 \%, p=0.003\right)$ was found. The results of this meta-analysis showed a potential protective effect of GT consumption on $\mathrm{BC}$, especially for $\mathrm{BC}$ recurrence.
\end{abstract}

Keywords: breast cancer risk; green tea; epigallocatechin; women; cancer recurrence; Camellia sinensis; meta-analysis

\section{Introduction}

Tea is one of the most consumed beverages worldwide. It is obtained from leaves and buds of Camellia sinensis (C. sinensis). The fruit of $C$. sinensis is three-celled and green in color, and the seed is a brown, semi-globose nut [1,2]. Young and light green leaves, as well as new shoots, are generally harvested to produce tea. After harvesting, various techniques of processing tea leaves lead to three main types of tea, characterized by different polyphenols content, from both a qualitative and quantitative point of view, and by different flavors. 
Green tea is produced by exposing fresh leaves to heat or hot steam immediately after plucking, and this results in minimal polyphenol oxidation. If fresh leaves are allowed to stand for an hour (or less) before heat treating, the final product is called oolong tea, characterized by an intermediate stage of oxidation between green and black tea. The latter is obtained by a series of processes, including withering, pre-conditioning, "cut-tear-curl", fermentation, where the leaves are exposed to the air, and final drying [3,4].

Initially, tea was traditionally used in a popular Chinese medicine, and only later did it spread as a beverage. Its history is intricate, and its origin is still uncertain. Some legends claim it was discovered in China by Emperor Shennong, around 5000 years ago; others affirm it originated in India and was later brought to China, Japan, and Korea thanks to the spread of Buddhism [5]. One of the earliest written references dates back to 221 B.C., when the Chinese Emperor Qin Shi Huang signed a document to introduce a tax on tea, while the first handbook was written by $\mathrm{Lu}-\mathrm{Yu}$ in the eighth century [6]. Tea was first introduced in the Western World by the Turks (around 600 AD), but only in the seventeenth century did it began to spread all over Europe, thanks to Dutch and English imports from Asia (India, Sri Lanka, China, Japan, Indonesia) [5-7]. Nowadays, tea is produced in several countries: the main producer is China (1.9 million metric tons in 2013), followed by India, Kenya, and Sri Lanka [8].

The potential beneficial effects of tea are due to the large amount of bioactive compounds (approximately 4000), one-third of which belong to the polyphenol group [7]. Catechins are the most important polyphenol class in tea. Tea also contains flavonols, phenolic acids, and methylxanthines $[9,10]$. Among teas, different production techniques translate into different phytochemical characteristics. Green tea is the most studied for its potential benefits, since it retains a major amount of catechins in comparison to other teas. Indeed, catechins constitute $30-42 \%$ of the total dry weight of green tea [10]. The most abundant catechins are (-)-epigallocatechin-3-gallate (EGCG), (-)-epigallocatechin (EGC), (-)-epicatechin-3-gallate (ECG), and (-)-epicatechin (EC), which represent approximately $59 \%, 19 \%, 13 \%$, and $6.4 \%$ of all catechins, respectively [11]. Several studies have demonstrated preventive and likely chemotherapeutic activities of green tea polyphenols and EGCG against breast, skin, colon, lung, prostate, liver, and stomach cancer [12-18]. However, the molecular mechanisms are still unknown. Among the most plausible mechanisms involved, antioxidant activity, inhibition of clonal expansion of cancer stem cells by the maintenance of a quiescent state, downregulation of oncogenes and upregulation of tumor-suppressor genes through epigenetic phenomena, regulation of telomerase activity, and modulation of membrane lipid raft have been investigated [19-22]. Recently, green tea EGCG has been shown to inhibit angiogenesis through different pathways and mechanisms such as modulation of cell proliferation, suppression of angiogenic factors, and induction of apoptosis [20,21]. Moreover, miRNAs might be involved in the process via the inhibition of binding of vascular endothelial growth factor (VEGF) to its receptor [23]. In particular, previous studies highlighted the role played by EGCG on epigenetic modification of genes involved in early carcinogenesis and/or breast cancer progression. Among females, breast cancer (BC) is the most frequent cancer, with the highest cancer mortality rate among women worldwide [24]. According to GLOBOCAN, in 2018, BC will represent almost 1 in 4 of cancer cases among women, with 2.1 million new diagnoses [24]. Moreover, BC incidence is higher in Europe and North America compared to Asia and Africa. Although well-known hereditary and genetic factors are involved in BC aetiology, the increased risk among low-risk populations migrating into high-risk areas revealed the potential role played by other factors such as oral contraceptive drugs, parity, menstruation, physical activity, breast feeding, and nutrition [25]. Previous studies provided limited/non-conclusive evidence on the correlation between green tea consumption and the risk of BC. The aim of this systematic review and meta-analysis was to evaluate the association between green tea regular consumption and risk of $\mathrm{BC}$ in women. Furthermore, the risk of $B C$ recurrence and risk of $B C$ in relation to menopausal status was assessed. 


\section{Material and Methods}

Preferred Reporting Items for Systematic Reviews and Meta-Analyses (PRISMA) guidelines were followed to perform the systematic review and meta-analysis [26]. A literature search was conducted on 18 September 2017 and updated on 19 November 2018 in PubMed, Scopus, and Web of Science. The databases were searched using appropriate key words, pre-determined based on the type of the database consulted. In PubMed/Medline, a combination of Medical Subject Headings (MeSH) terms, title/abstract, and all field were used. In both databases, the search terms were selected based on three aspects: green tea consumption, breast cancer risk, and type of study. The keywords were then combined using Boolean operator AND/OR. Considering the listed aspects, the search strategy for PubMed/Medline was:

-green tea consumption: "green tea"[Title/Abstract] OR "Camellia sinensis"[MeSH] OR "Tea"[MeSH] OR "green tea"[Title/Abstract] OR "Polyphenols"[MeSH] OR "epigallocatechin gallate"[Supplementary Concept];

-breast cancer risk: (("breast neoplasms"[MeSH Terms] OR breast cancer[Text Word] OR breast cancer[Title/Abstract] OR ((“breast”[MeSH Terms] OR “breast”[All Fields]) AND tumor[Title/Abstract];

-study design: “Case-Control Studies"[MeSH] OR “Cohort Studies"[MeSH] OR “Retrospective Studies"[MeSH] OR "Prospective Studies"[MeSH].

For searches on Scopus, the following search terms were used:

-green tea consumption: "green tea" OR “Camellia sinensis" OR Tea OR epigallocatechin gallate

OR Polyphenols

-breast cancer risk: breast neoplasm OR breast cancer OR breast tumor

-study design: "Case-Control Studies" OR “Cohort Studies" OR “Retrospective Studies" OR "Prospective Studies".

\subsection{Inclusion Criteria}

Articles included in the current meta-analysis met the following criteria: studies performed on humans, research focused on green tea consumption, full text available, epidemiologic studies, only articles in English. Inversely, exclusion criteria were: studies with different outcomes, data not reported as risk (for instance Odds Ratio - OR; Relative Risk - RR; Hazard Ratio - HR) or articles without original data, and animal model trials. As the aim of this review was not to evaluate the effectiveness of an intervention in a population, randomized controlled trials (RCTs) were not included [27]. Furthermore, green tea drinking is a common human practice so it is difficult to evaluate by experimental study design. Indeed, all the RCTs retrieved during the reference screening evaluated the effect of green tea extract supplementation instead of green tea consumption. Details of inclusion/exclusion criteria are presented in Table 1, according to PICOS (Population, Intervention, Comparison, Outcome, Study type) format expanded with time filter and language [28].

Table 1. Search strategy details.

\begin{tabular}{|c|c|}
\hline Search Strategy & Details \\
\hline Inclusion criteria & $\begin{array}{l}\text { P: general adult population (male and female) } \\
\text { I: questionnaire or interview assessing green tea consumption } \\
\text { C: higher vs. lower green tea consumption } \\
\text { O: risk of breast cancer (if any) } \\
\text { S: original research article (case-control studies, cohort studies, cross-sectional studies) }\end{array}$ \\
\hline Exclusion criteria & $\begin{array}{l}\text { P: pediatric population } \\
\text { I: no administration of questionnaire } \\
\text { O: other outcomes } \\
\text { S: review article, expert opinion, comments, abstract, letters, article with no quantitative } \\
\text { information or details }\end{array}$ \\
\hline Language filter & English \\
\hline Time filter & None (from inception) \\
\hline
\end{tabular}


Table 1. Cont.

\begin{tabular}{ll}
\hline Search Strategy & Details \\
\hline Database & PubMed/Medline; Scopus; Web of Science \\
\hline Abbreviations: P = Population; I = Intervention; C = Comparison; O = Outcome; S = Studies.
\end{tabular}

To validate the inclusion of the retrieved studies, two reviewers (V.G. and A.A.), independently and blinded, screened titles and abstracts. Possible disagreements were resolved through discussion or third reviewer consultation (D.N.). The reviewers read the full texts of the selected articles and double-checked the list of references of the selected studies in order to detect any other potentially relevant papers.

\subsection{Data Extraction}

Data were collected independently by two authors (V.G. and A.A.) and reported in a pre-determined spreadsheet. Extracted data contained both qualitative and quantitative information. In particular, qualitative data included the name of the first author, year of publication, type of study, and country where the study was conducted. Quantitative study design characteristics, namely data on enrolment, exposure, tools used to estimate food intake (for instance, Food Frequency Questionnaire (FFQ), validated or not, with the FFQ either self-administered or by interview), and information on the outcomes, were also collected. Furthermore, participant characteristics such as sample size, age range or mean age, and health status were also collected.

\subsection{Quality Evaluation}

In order to perform a sensitivity analysis, the quality of the included studies was also evaluated by two authors (A.A. and M.A.) using the scoring system created on the basis of Meta-analysis of Observational Studies in Epidemiology (MOOSE) [27], Quality Assessment Tool for Systematic reviews of Observational studies (QATSO) [29], and STrengthening the Reporting of OBservational studies in Epidemiology (STROBE) [30] and modified by Buitrago-Lopez et al., who applied the scoring tool on food intake and risk of chronic diseases [31]. The scoring system assigns zero or one point based on the evaluation of the six variables considered. The highest score available is six and the variables analyzed are: (i) justification given for the cohort (at least $80 \%$ of the initial included participants); (ii) appropriate inclusion and exclusion criteria; (iii) histological diagnosis of breast cancer; (iv) validated tool to assess green tea consumption; $(v)$ adjustments were made for age, sex, body mass index, smoking status, and parity; and (vi) any other adjustments (such as for dietary factors, age at menarche, menopausal status, alcohol consumption).

\subsection{Statistical Analysis}

The software ProMeta 3 (Internovi, Cesena, Italy) was used to run the meta-analysis. The effect size (ES) was estimated by odds ratio (OR) reported with its $95 \%$ confidence interval (CI). For this study, $p<0.05$ was considered statistically significant. The statistical heterogeneity among studies was assessed by the $\mathrm{Chi}^{2}$ test and $I^{2}$ statistic (considering a value $I^{2}>60 \%$ as being statistically heterogeneous) [32]. To calculate the pooled effect, a fixed effect model was applied according to the found heterogeneity (Egger's linear regression test). Lastly, a funnel plot was visually evaluated to assess possible publication bias. In addition, the plot of publication year bias was also evaluated.

The meta-analysis was conducted considering the risk of breast cancer among participants exposed to the highest versus the lowest consumption of green tea, as reporting of the frequency, dose, and unit (mL, g, cups, etc.) of tea consumption varied among the original studies.

\subsection{Sensitivity Analysis}

In order to corroborate the strength of the results, an additional subgroup analysis was performed, taking into account quality score, study design, risk of $\mathrm{BC}$, and recurrence. 


\section{Results}

\subsection{Literature Search}

From PubMed/Medline, 47 articles were retrieved, whilst 56 articles were found in Scopus and 90 in Web of Science. After bibliography list screening, one more article was added, giving a total of 194 potentially relevant articles. However, 39 studies were duplicates and immediately eliminated. After further screening, based on evaluation of title and abstract, another 115 records were excluded, as reported in Figure 1. Full texts were downloaded for the remaining 40 studies. A further 25 studies were excluded for various reasons, mainly because no differentiation between green tea and other teas was taken into account (see Table S1 in the Supplementary Materials) [33-57]. The remaining 15 studies were included for quality synthesis. Nevertheless, two other studies were eliminated because data were not extrapolated $[58,59]$. At the end of the screening flow, 13 records were included in the meta-analysis. However, because Suzuki et al. reported the risk of BC in two different cohorts, this paper was considered as two independent studies [60].

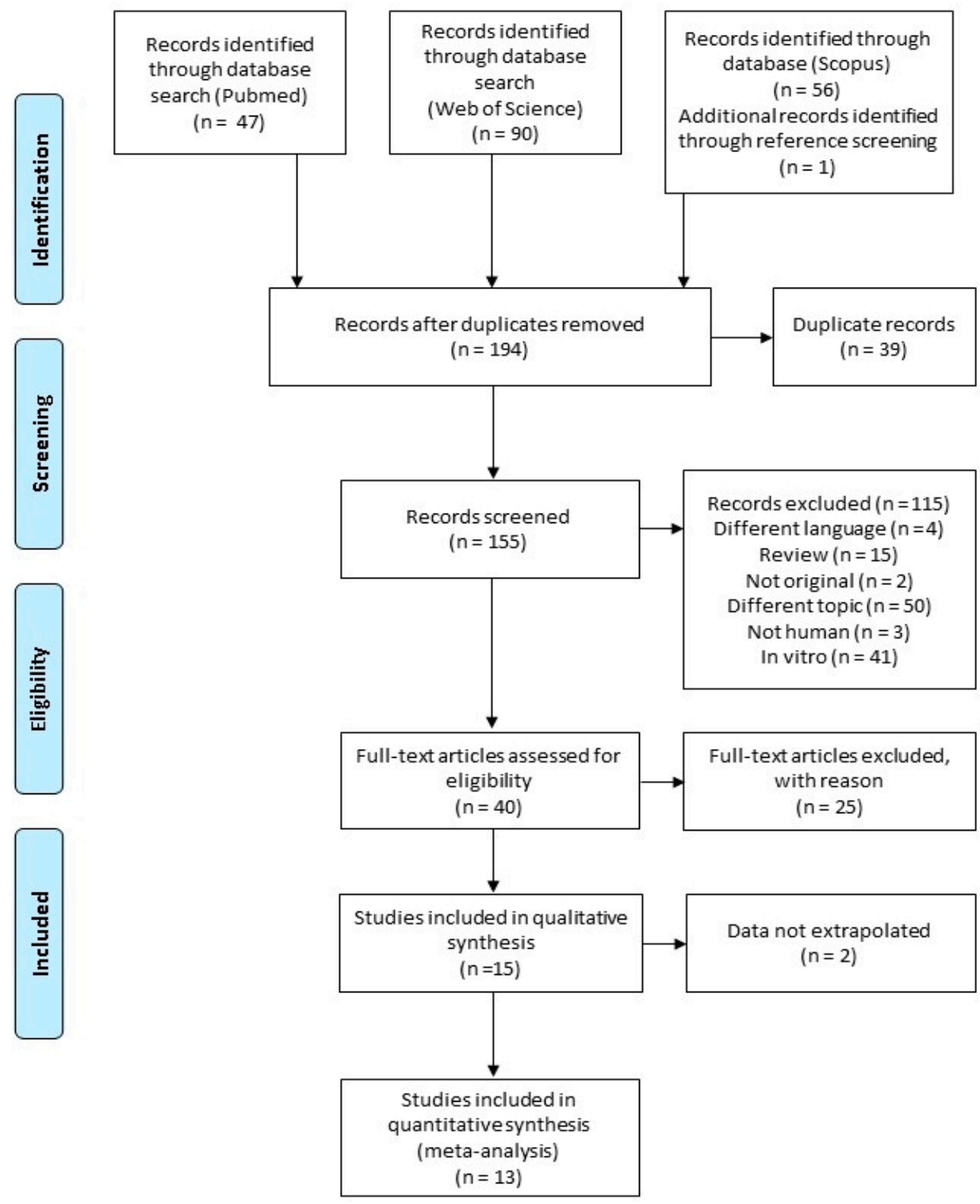

Figure 1. Flow diagram of studies selection process. 


\subsection{Characteristics of Included Studies}

All the studies included in the analysis are tabulated in Table 2. Looking at the country where the studies were performed, seven studies were conducted in Japan [60-66], five in China [67-71], and only one in the United States of America (USA) [72]. The age of the participants ranged between 20-87 years. Moreover, almost all the studies were relatively recent; only two studies were conducted before $2000[64,66]$. Around half of the studies were cohort studies $[60-62,64-67,70]$ and five were case-control studies $[63,68,69,71,72]$. Moreover, seven studies were conducted on women with diagnosis of $B C$ analyzing the risk of $B C$ recurrence $[61,63,66,68,69,71,72]$, whilst six studies recruited healthy participants and estimated the risk of $B C[60,62,64,65,67,70]$. The pooled sample consisted of $163,810$ people and the ES was $0.85((95 \% \mathrm{CI}=0.80-0.92), p=0.000))$ (Figure $2 \mathrm{a})$. The results of this meta-analysis show an important effect in the highest vs. the lowest category of green tea consumption; moreover, Egger's linear regression test does not show a potential publication bias (intercept 0.33, $t=0.40, p=0.695$ ), also confirmed by the symmetry of the funnel plot (Figure $2 b$ ). No high statistical heterogeneity $\left(\mathrm{Chi}^{2}=31.55, d f=13, I^{2}=58.79 \%, p=0.003\right)$ was found. Figure $2 \mathrm{c}$ shows the potential bias due to year of publication. 
Table 2. Characteristics extracted from the included studies and quality score.

\begin{tabular}{|c|c|c|c|c|c|c|c|c|c|c|c|c|}
\hline Author, Year & No in Analysis & Age (years) & Baseline & $\begin{array}{l}\text { Study } \\
\text { Period }\end{array}$ & $\begin{array}{c}\text { Study } \\
\text { Design }\end{array}$ & Instrument & Outcome & $\begin{array}{c}\text { Green Tea } \\
\text { Intake }\end{array}$ & $\begin{array}{c}\text { OR RR HR } \\
\text { (CI 95\%) }\end{array}$ & $p$-Value & Country & $\mathrm{QS} / 6$ \\
\hline Li M. et al. 2016 & $\begin{array}{l}\text { Case }=756 \\
\text { Control }=789\end{array}$ & $20-84$ y & $\begin{array}{l}\text { BC diagnosis } \\
\text { All stage }\end{array}$ & 2011-2014 & Case-control & $\begin{array}{l}\text { In-person } \\
\text { interview }\end{array}$ & recurrence & 3 cups/day & $\begin{array}{c}1.2 \\
(0.8-1.78)\end{array}$ & 0.38 & China & 4 \\
\hline $\begin{array}{l}\text { Iwasaki M. et al. } \\
2014\end{array}$ & $\begin{array}{l}\text { Case }=369 \\
\text { Control }=369\end{array}$ & $20-74$ y & $\begin{array}{c}\text { BC diagnosis } \\
1-2 \text { stage }\end{array}$ & 2001-2005 & Case-control & FFQ validated & recurrence & $600 \mathrm{~mL} /$ day & $\begin{array}{c}1.27 \\
(0.75-2.14) \\
\end{array}$ & 0.20 & Japan & 5 \\
\hline $\begin{array}{l}\text { Iwasaki M. et al. } \\
2010\end{array}$ & 67,422 & $40-69 y$ & $\begin{array}{l}\text { Healthy } \\
\text { women }\end{array}$ & $\begin{array}{l}\text { 1990-1994 } \\
\text { Follow-up } \\
\text { 1995-1998 }\end{array}$ & $\begin{array}{l}\text { Prospective } \\
\text { cohort }\end{array}$ & $\begin{array}{c}\mathrm{FFQ} \\
\text { (self-administered) } \\
\text { not validated }\end{array}$ & BC & 5 cups/day & $\begin{array}{c}1.12 \\
(0.81-1.56)\end{array}$ & 0.60 & Japan & 5 \\
\hline $\begin{array}{l}\text { Shrubsole J. M. } \\
\text { et al. } 2009\end{array}$ & $\begin{array}{l}\text { Case }=3454 \\
\text { Control }=3474\end{array}$ & $25-70 y$ & BC diagnosis & 1996-2005 & Case-control & FFQ validated & recurrence & $\begin{array}{c}148 \pm 124 \\
\mathrm{~g} / \mathrm{mo}\end{array}$ & $\begin{array}{c}0.88 \\
(0.79-0.98) \\
\end{array}$ & n.a. & China & 4 \\
\hline $\begin{array}{l}\text { Inoue M. et al. } \\
2008\end{array}$ & 678 & $45-74$ y & $\begin{array}{l}\text { Healthy } \\
\text { women }\end{array}$ & 1993-1998 & $\begin{array}{c}\text { Prospective } \\
\text { cohort }\end{array}$ & $\begin{array}{l}24 \mathrm{~h} \text { food recalls } \\
\text { validated }\end{array}$ & BC & $\begin{array}{c}174.6 \pm 75.2 \\
\mu \mathrm{g} / \text { day }\end{array}$ & $\begin{array}{c}1.00 \\
(0.82-1.22) \\
\end{array}$ & 0.41 & China & 4 \\
\hline $\begin{array}{l}\text { Zhang M. et al. } \\
2007\end{array}$ & $\begin{array}{l}\text { Case }=1009 \\
\text { Control = } 1009\end{array}$ & $20-87$ y & $\mathrm{BC}$ diagnosis & 2004-2005 & Case-control & FFQ validated & recurrence & 4 cups/day & $\begin{array}{c}0.57 \\
(0.47-0.69)\end{array}$ & 0.001 & China & 6 \\
\hline $\begin{array}{l}\text { Yuan J.M. et al. } \\
2005\end{array}$ & 665 & $45-74$ y & $\begin{array}{l}\text { Healthy } \\
\text { women }\end{array}$ & 1993-1998 & $\begin{array}{l}\text { Prospective } \\
\text { cohort }\end{array}$ & $\begin{array}{l}\text { In-person } \\
\text { interview }\end{array}$ & BC & Weekly & $\begin{array}{c}0.91 \\
(0.66-1.26)\end{array}$ & n.a. & China & 4 \\
\hline $\begin{array}{l}\text { Suzuki Y. et al. } \\
2004 \text { (a) }\end{array}$ & 17,353 & $\begin{array}{c}>40 \mathrm{y} \\
\text { (cohort I) }\end{array}$ & $\begin{array}{l}\text { Healthy } \\
\text { women }\end{array}$ & 1984-1990 & $\begin{array}{c}\text { Prospective } \\
\text { cohort }\end{array}$ & $\begin{array}{c}\text { FFQ validated } \\
\text { (self-administered) }\end{array}$ & BC & 5 cups/day & $\begin{array}{c}0.96 \\
(0.50-1.86) \\
\end{array}$ & 0.51 & Japan & 6 \\
\hline $\begin{array}{l}\text { Suzuki Y. et al. } \\
2004 \text { (b) }\end{array}$ & 24,769 & $\begin{array}{c}40-64 \mathrm{y} \\
\text { (cohort II) }\end{array}$ & $\begin{array}{l}\text { Healthy } \\
\text { women }\end{array}$ & 1984-1990 & $\begin{array}{c}\begin{array}{c}\text { Prospective } \\
\text { cohort }\end{array} \\
\end{array}$ & $\begin{array}{c}\text { FFQ validated } \\
\text { (self-administered) }\end{array}$ & BC & 5 cups/day & $\begin{array}{c}0.85 \\
(0.43-1.669) \\
\end{array}$ & 0.95 & Japan & 6 \\
\hline $\begin{array}{l}\text { Wu H. A. et al. } \\
2003\end{array}$ & $\begin{array}{l}\text { Case }=501 \\
\text { Control }=594\end{array}$ & $25-74$ y & BC diagnosis & 1995-1998 & Case-control & $\begin{array}{l}\text { In-person } \\
\text { interview }\end{array}$ & recurrence & $85.7 \mathrm{~mL} /$ day & $\begin{array}{c}0.61 \\
(0.40-0.93) \\
\end{array}$ & 0.01 & USA & 4 \\
\hline $\begin{array}{l}\text { Inoue M. et al. } \\
2001\end{array}$ & 1160 & $\begin{array}{c}\text { Mean age } \\
51.5 \mathrm{y}\end{array}$ & $\begin{array}{l}\text { BC diagnosis } \\
1-2 \text { stage }\end{array}$ & 1990-1997 & Follow-up & $\begin{array}{c}\mathrm{FFQ} \\
\text { (self-administered) } \\
\text { not validated }\end{array}$ & recurrence & 6 cups/day & $\begin{array}{c}0.68 \\
(0.4-1.16)\end{array}$ & 0.72 & Japan & 3 \\
\hline $\begin{array}{l}\text { Nagano J. et al. } \\
2001\end{array}$ & 38,540 & $\begin{array}{c}\text { Mean age } \\
54.8 \mathrm{y}\end{array}$ & $\begin{array}{l}\text { Healthy } \\
\text { women }\end{array}$ & 1979-1981 & $\begin{array}{l}\text { Prospective } \\
\text { cohort }\end{array}$ & $\begin{array}{c}\mathrm{FFQ} \\
\text { (self-administered) } \\
\text { not validated }\end{array}$ & $\begin{array}{l}\text { BC and other } \\
\text { cancers }\end{array}$ & 5 cups/day & $\begin{array}{c}1.0 \\
(0.67-1.6)\end{array}$ & 0.80 & Japan & 3 \\
\hline $\begin{array}{l}\text { Key T.J. et al. } \\
1999\end{array}$ & 34,759 & $40-80 y$ & $\begin{array}{l}\text { Healthy } \\
\text { women }\end{array}$ & 1969-1981 & $\begin{array}{c}\text { Prospective } \\
\text { cohort }\end{array}$ & $\begin{array}{c}\text { FFQ not } \\
\text { validated }\end{array}$ & BC & 5 cups/day & $\begin{array}{c}0.86 \\
(0.62-1.21) \\
\end{array}$ & 0.284 & Japan & 3 \\
\hline $\begin{array}{l}\text { Nakachi K. et al. } \\
1998\end{array}$ & 472 & $\begin{array}{l}\text { Mean age } \\
49.7 \pm 11.2\end{array}$ & $\begin{array}{c}\text { BC diagnosis } \\
1-2-3 \text { stage }\end{array}$ & 1984-1993 & Follow-up & $\begin{array}{c}\text { FFQ not } \\
\text { validated }\end{array}$ & recurrence & 8 cups/day & $\begin{array}{c}0.775 \\
(0.53-1.13)\end{array}$ & 0.15 & Japan & 4 \\
\hline
\end{tabular}

Abbreviations: QS = quality score; $\mathrm{BC}=$ breast cancer; $\mathrm{FFQ}$ = food frequency questionnaire; .. . $=$ not available; OR = odds ratio; RR = Relative Risk; HR = Hazard Ratio 
a)

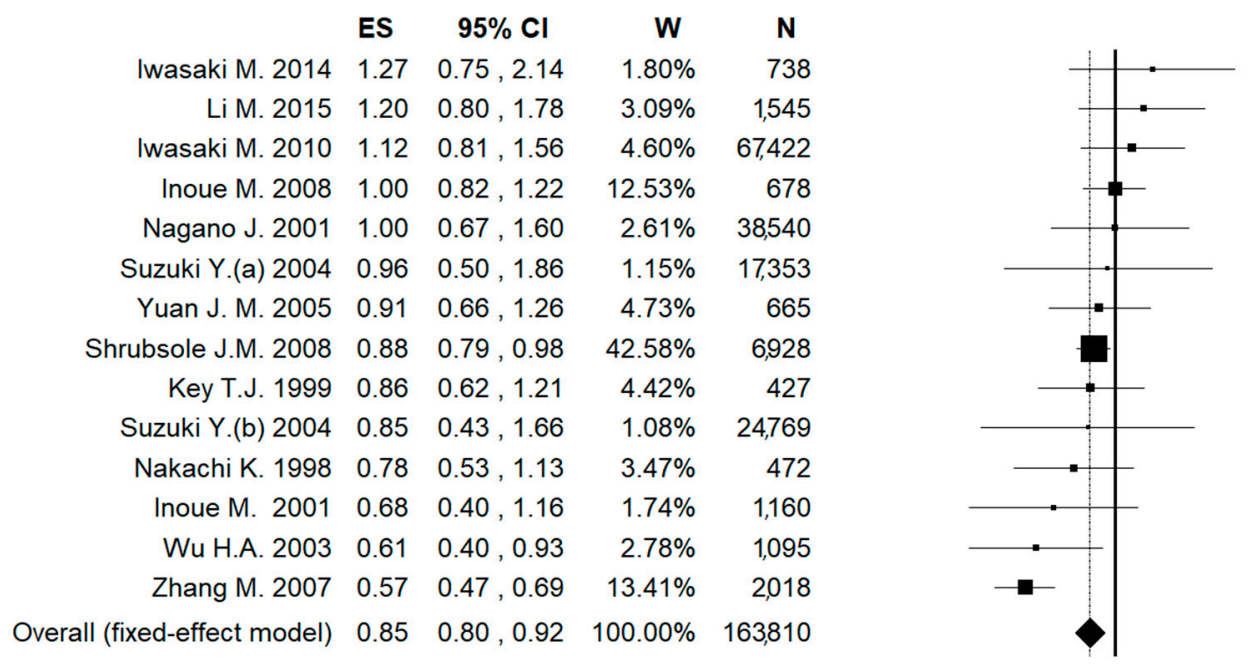

b)

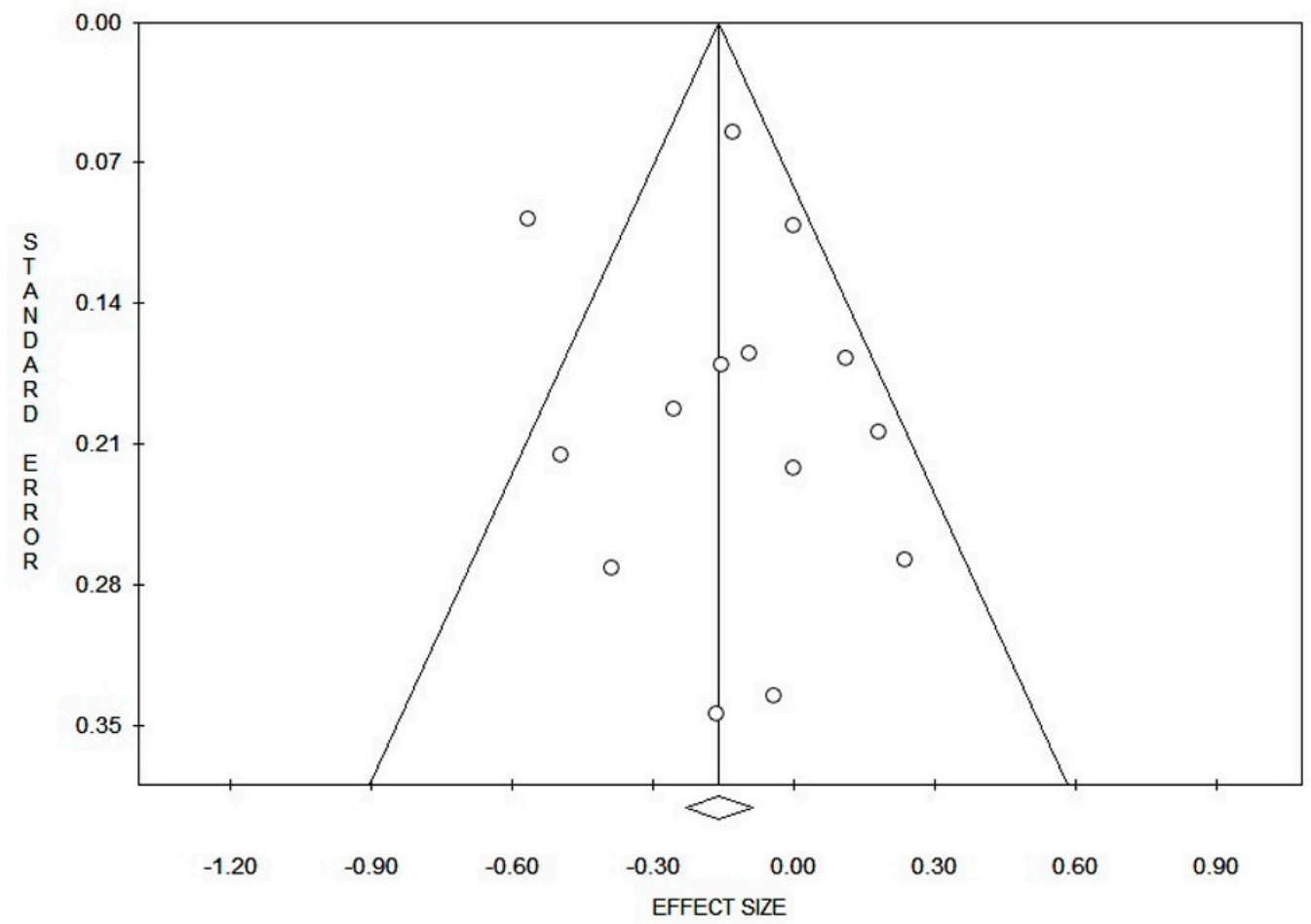

Figure 2. Cont. 


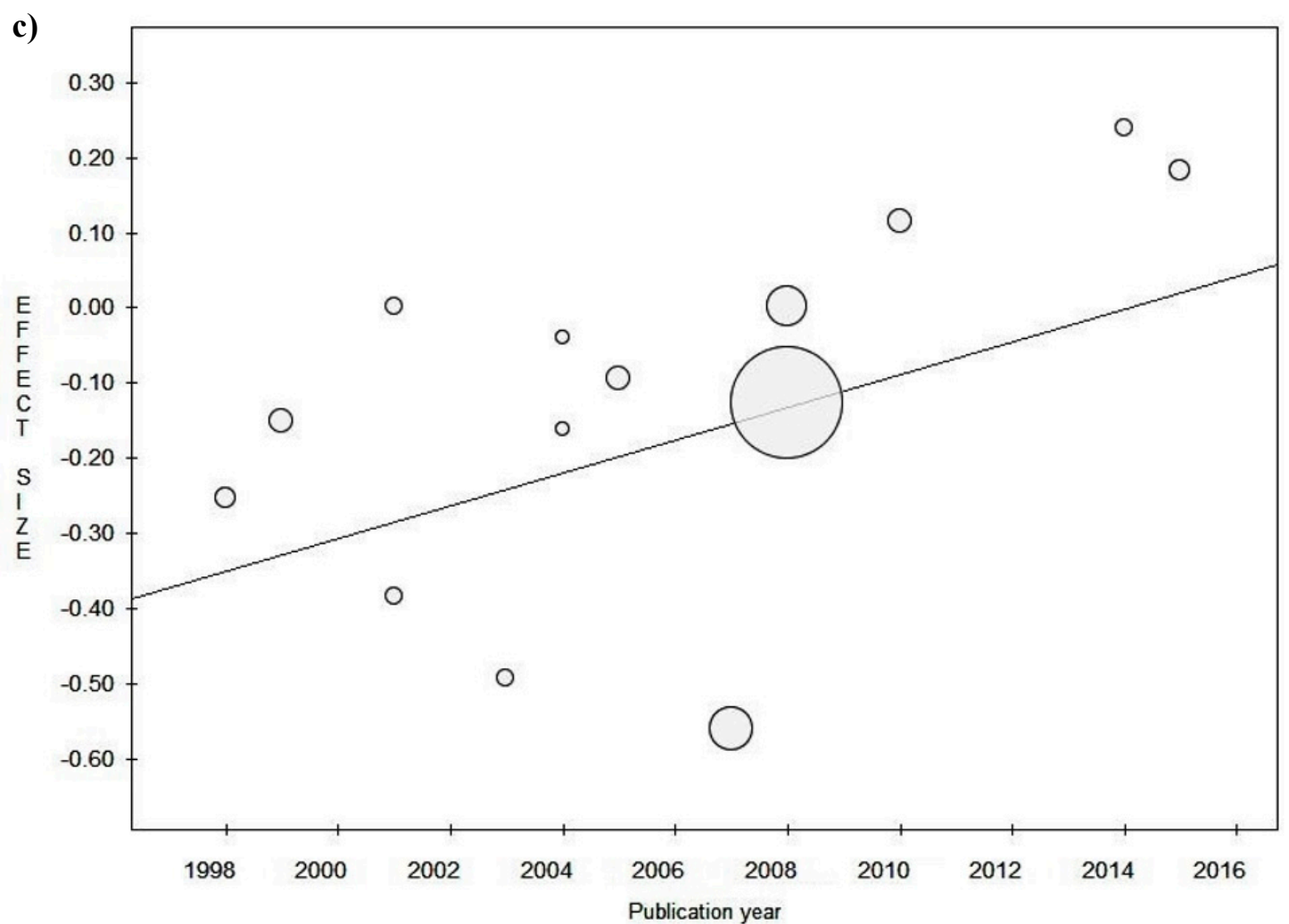

Figure 2. Forest plot (a) of the meta-analysis comparing green tea consumption in breast cancer (BC). Funnel plot (b). Publication year plot (c). Abbreviations: $\mathrm{ES}=$ effect size; $\mathrm{CI}=$ confidence interval; $\mathrm{W}=$ weight; $\mathrm{Sig}=$ significance; $\mathrm{N}=$ number.

\subsection{Sensitivity Analysis}

\section{Study Design}

Any potential change in the results was analyzed, firstly performing the analysis according to the original studies design. When only cohort studies were included in the meta-analysis, the selection ranged from 472 to 67,422 participants followed up for 6 years on average. All the studies recruited healthy participants except for two papers that selected women with BC diagnosis. Considering all the cohort studies, the pooled sample size consisted of 151,486 participants with ES $=0.94((95 \%$ $\mathrm{CI}=0.83-1.05), p=0.273$ ) ) and with low potential publication bias (intercept $-0.90, t=-1.38, p=0.209$, statistical heterogeneity $\left.\mathrm{Chi}^{2}=4.3, d f=8, p=0.822, \mathrm{I}^{2}=0.00, \mathrm{~T}^{2}=0.00\right)$. However, when the two studies that enrolled women with BC were excluded, the results did not change significantly ES $=0.97((95 \%$ $\mathrm{CI}=0.86-1.11), p=0.273)$ ) (intercept $-0.38, t=-0.69, p=0.521$, statistical heterogeneity $\mathrm{Chi}^{2}=1.64$, $\left.d f=6, p=0.950, I^{2}=0.00, T^{2}=0.00\right)$. Inversely, after selective inclusion of only case-control studies, the pooled sample size consisted of 12,324 participants with ES $=0.81([(95 \% \mathrm{CI}=0.74-0.89), p=0.000))$ and with no potential publication bias (intercept $0.12, t=0.05, p=0.962$ ), even though a statistical heterogeneity was found $\left(\mathrm{Chi}^{2}=23.42, d f=4, p=0.000, I^{2}=82.92, T^{2}=0.08\right)$; probably due to a low number of included studies. Data are shown in Figure $3 a$ (forest plot) and $3 b$ (funnel plot). 
a)

$\begin{array}{rccrcc} & \text { ES } & \mathbf{9 5 \%} \text { Cl } & \text { W } & \text { Sig. } & \text { N } \\ \text { Iwasaki M. 2014 } & 1.27 & 0.75,2.14 & 2.83 \% & 0.372 & 738 \\ \text { Li M. 2016 } & 1.20 & 0.80,1.78 & 4.86 \% & 0.372 & 1,545 \\ \text { Shrubsole J.M. 2009 } & 0.88 & 0.79,0.98 & 66.88 \% & 0.020 & 6,928 \\ \text { Wu H.A. 2003 } & 0.61 & 0.40,0.93 & 4.36 \% & 0.022 & 1,095 \\ \text { Zhang M. 2007 } & 0.57 & 0.47,0.69 & 21.07 \% & 0.000 & 2,018 \\ \text { Overall (fixed-effect model) } & 0.81 & 0.74,0.89 & 100.00 \% & 0.000 & 12,324\end{array}$

b)

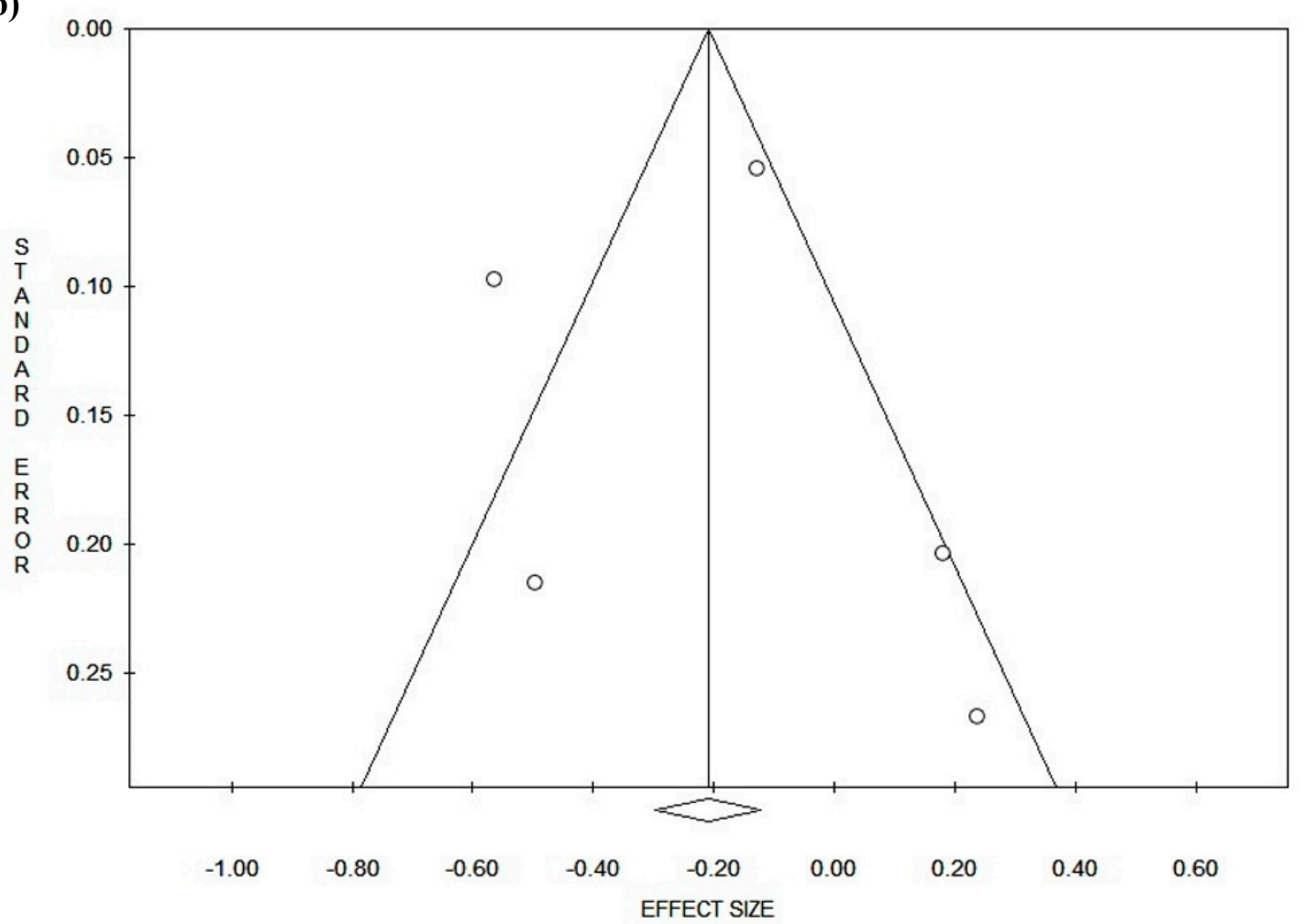

Figure 3. Forest plot (a) of the meta-analysis comparing green tea consumption in breast cancer (BC) prevention (case-control studies). Funnel plot (b). Abbreviations: ES = effect size; $\mathrm{CI}=$ confidence interval; $\mathrm{W}$ = weight; Sig = significance; $\mathrm{N}$ = number.

\subsection{Outcome: BC Diagnosis and BC Recurrences}

Seven studies focused on the risk of BC recurrence $[61,63,66,68,69,71,72]$ : one was conducted in USA, three in China, and the remaining in Japan. In three of these studies, the ESs indicated a potential protective effect of $\mathrm{BC}$ recurrence, while in the other four papers, the reduction was not statistically significant. The pooled ES was $0.81((95 \% \mathrm{CI}=0.74-0.88), p$-value $=0.000)$ based on a total of 13,956 participants, with participant numbers in individual studies ranging from 472 to 6928 participants (Figure 4a). A slight statistical heterogeneity was found $\left(\mathrm{Chi}^{2}=23.87, d f=6, \mathrm{I}^{2}=74.56 \%, p=0.001\right)$. The funnel plot (Figure $4 \mathrm{~b}$ ) shows no potential publication bias, which was confirmed by Egger's linear regression test (Intercept $-0.16, t=-0.11, p=0.915$ ).

A supplementary analysis of the studies focusing on the risk of new BC diagnosis was conducted. In this case, none of the included studies reported a statistically significant ES, which was also confirmed by the pooled ES of the current meta-analysis. Indeed, the combined ES was $0.97((95 \%$ $\mathrm{CI}=0.86-1.11), p$-value $=0.684)$. However, no statistical heterogeneity $\left(\mathrm{Chi}^{2}=1.64, d f=6, I^{2}=0.00 \%\right.$, 
$p=0.950$ ) was found (data not shown). Moreover, only three studies reported the stage of BC. In particular, Inoue et al. 2001 and Iwasaki et al. 2014 [63] only included women in the first and second stage of BC. In this analysis, ES was $1.0((95 \% \mathrm{CI}=0.90-1.10), p=0.926)$ based on 1898 , participants with no statistical heterogeneity $\left(\mathrm{Chi}^{2}=2.69, p=0.101, \mathrm{I}^{2}=62.76 \%\right)$.

a)

$\begin{array}{rccccc} & \text { ES } & \text { 95\% Cl } & \text { W } & \text { Sig. } & \text { N } \\ \text { Iwasaki M. 2014 } & 1.27 & 0.75,2.14 & 2.61 \% & 0.372 & 738 \\ \text { Li M. 2015 } & 1.20 & 0.80,1.78 & 4.49 \% & 0.372 & 1,545 \\ \text { Shrubsole J.M. 2008 } & 0.88 & 0.79,0.98 & 61.82 \% & 0.020 & 6,928 \\ \text { Nakachi K. 1998 } & 0.78 & 0.53,1.13 & 5.03 \% & 0.186 & 472 \\ \text { Inoue M. 2001 } & 0.68 & 0.40,1.16 & 2.53 \% & 0.156 & 1,160 \\ \text { Wu H.A. 2003 } & 0.61 & 0.40,0.93 & 4.03 \% & 0.022 & 1,095 \\ \text { Zhang M. 2007 } & 0.57 & 0.47,0.69 & 19.48 \% & 0.000 & 2,018 \\ \text { Il (fixed-effect model) } & 0.81 & 0.74,0.88 & 100.00 \% & 0.000 & 13,956\end{array}$

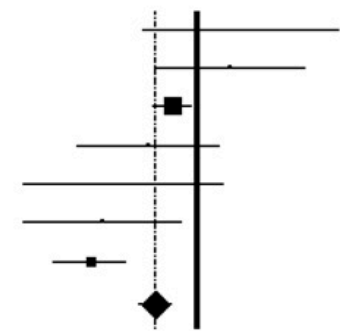

b)

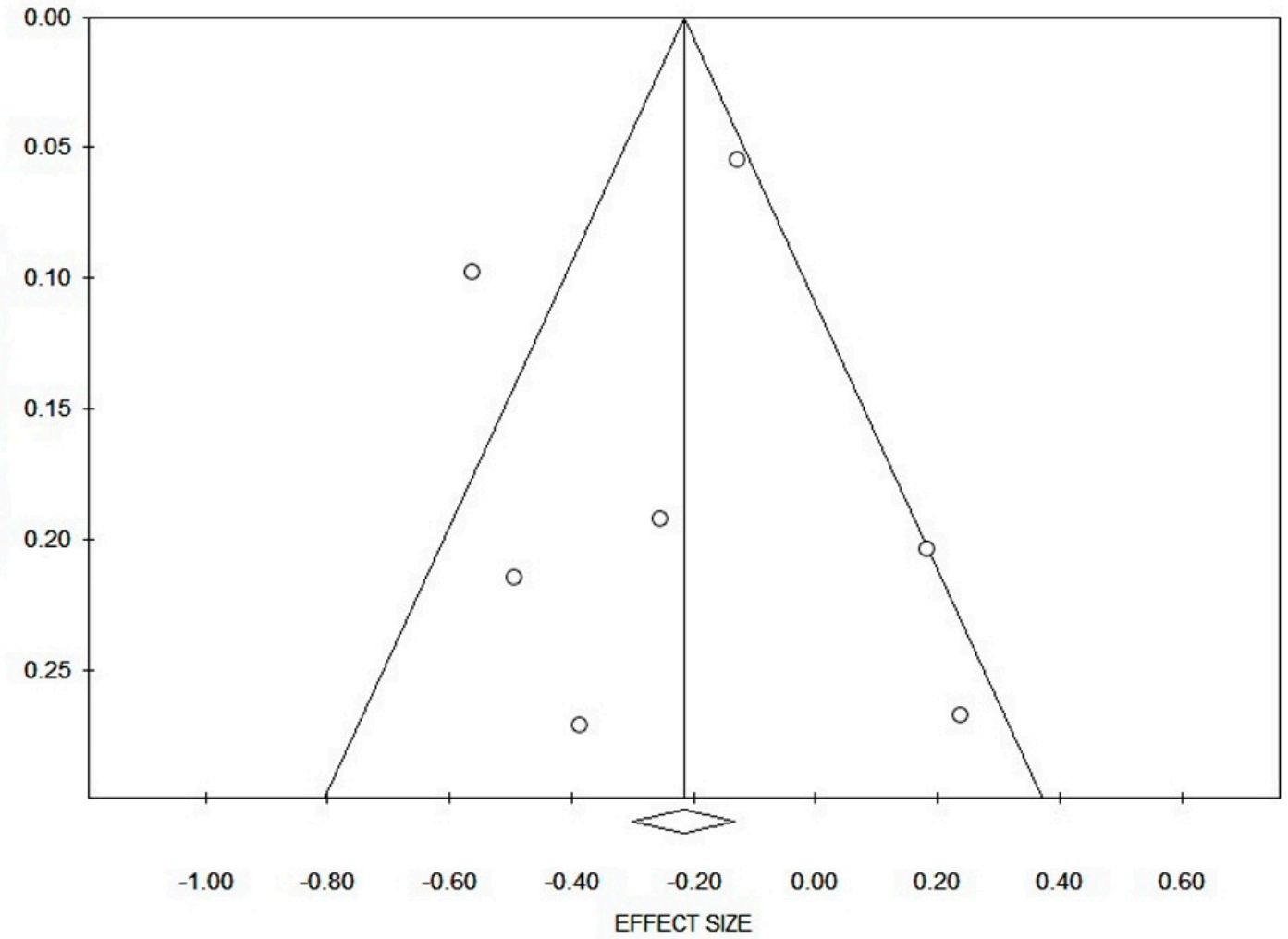

Figure 4. Forest plot (a) of the meta-analysis comparing green tea consumption in breast cancer (BC) recurrence. Funnel plot $(\mathbf{b})$. Abbreviations: $\mathrm{ES}=$ effect size; $\mathrm{CI}=$ confidence interval; $\mathrm{W}=$ weight; Sig = significance; $\mathrm{N}=$ number.

\subsection{Quality Score}

For a more comprehensive evaluation, a meta-analysis of studies with QS $\geq 5$ was performed. In this case, ES was $0.73((95 \% \mathrm{CI}=0.63-0.85), p$-value $=0.000)$ based on a total of 112,300 participants, with the number of participants for individual studies ranging from 738 to 67,422 participants (Figure $5 \mathrm{a}$ ). A slight statistical heterogeneity was found $\left(\mathrm{Chi}^{2}=18.08, d f=4, I^{2}=77.87 \%, p=0.001\right)$. The funnel plot (Figure $5 \mathrm{~b}$ ) shows potential publication bias, which was confirmed by Egger's linear regression test (intercept 2.90, $t=1.79, p=0.172$ ). 
a)

$\begin{array}{rcrrrr} & \text { ES } & 95 \% \text { Cl } & \text { W } & \text { Sig. } & \text { N } \\ \text { Iwasaki M. 2014 } & 1.27 & 0.75,2.14 & 8.16 \% & 0.372 & 738 \\ \text { Iwasaki M. } 2010 & 1.12 & 0.81,1.56 & 20.88 \% & 0.498 & 67,422 \\ \text { Suzuki Y.(a) } 2004 & 0.96 & 0.50,1.86 & 5.20 \% & 0.903 & 17,353 \\ \text { Suzuki Y.(b) } 2004 & 0.85 & 0.43,1.66 & 4.92 \% & 0.637 & 24,769 \\ \text { Zhang M. 2007 } & 0.57 & 0.47,0.69 & 60.84 \% & 0.000 & 2,018 \\ \text { Overall (fixed-effect model) } & 0.73 & 0.63,0.85 & 100.00 \% & 0.000 & 112,300\end{array}$

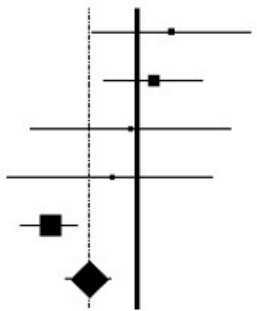

b)

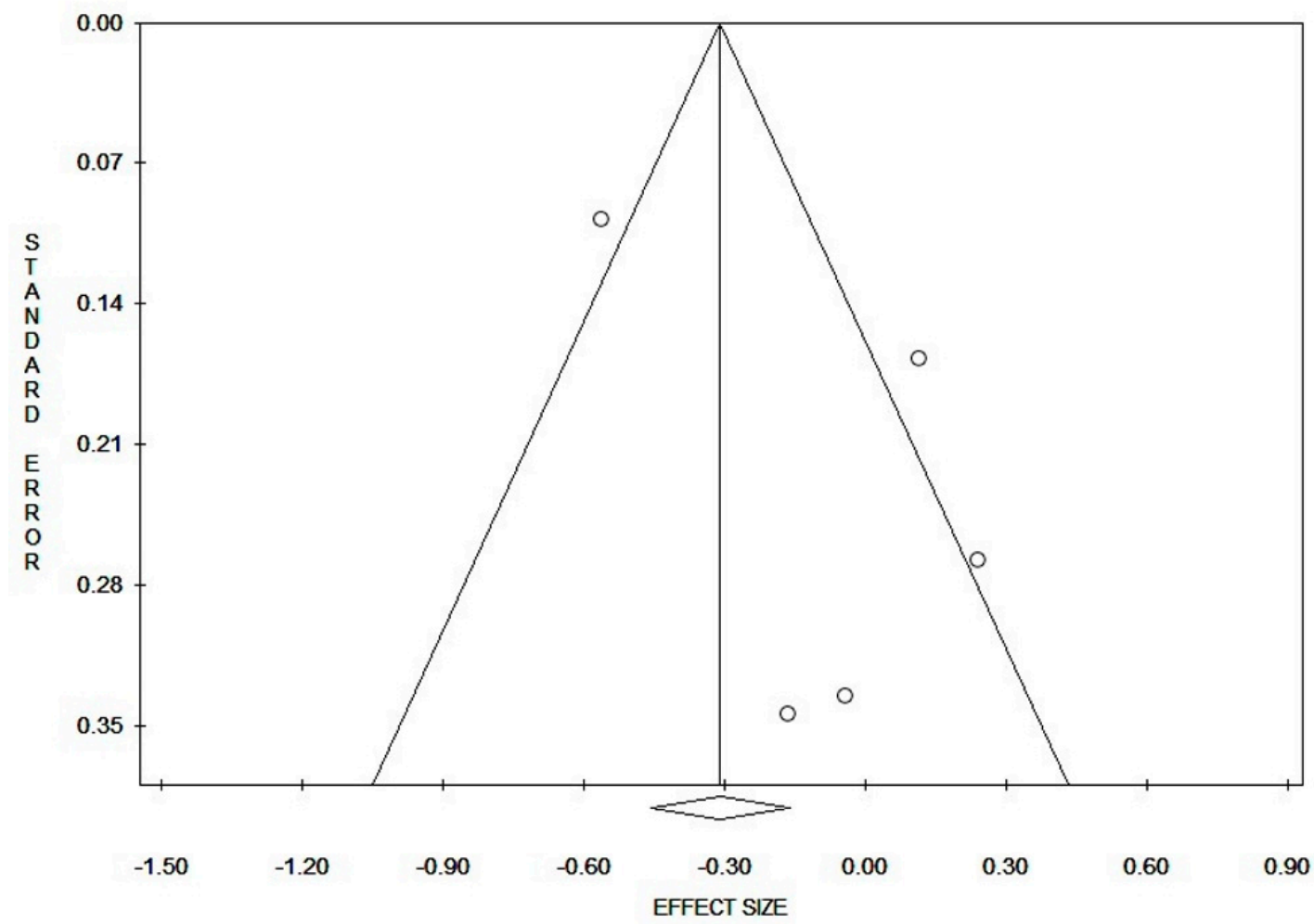

Figure 5. Forest plot (a) of the meta-analysis comparing green tea consumption in breast cancer (BC) (studies with quality score $\geq 5$ ). Funnel plot (b). Abbreviations: $E S=$ effect size; $C I=$ confidence interval; $\mathrm{W}$ = weight; $\mathrm{Sig}$ = significance; $\mathrm{N}$ = number.

\subsection{Amount of Green Tea Intake}

In order to find a potential beneficial amount of green tea, another meta-analysis of the studies reporting 5 cups of tea/day was performed. In this case, ES was 0.97 ( $(95 \% \mathrm{CI}=0.81-1.18)$, $p$-value $=0.783$ ) based on a total of 148,511 participants, with the number of participants for individual studies ranging from 427 to 67,422 participants. No statistical heterogeneity was found $\left(\mathrm{Chi}^{2}=1.46\right.$, $d f=5, I^{2}=0.00 \%, p=0.917$ ), and the lack of potential publication bias was confirmed by Egger's linear regression test (Intercept $=-0.23, t=-0.62, p=0.571$ ).

\subsection{Menopausal Status}

A meta-analysis looking at menopausal status was also performed (Table 3).

The risk of $\mathrm{BC}$ in women before and after menopause was compared. In the analysis, green tea intake showed a statistically significant protective role in pre-menopausal women. In this case, 
ES was $0.88((95 \% \mathrm{CI}=0.78-0.99), p$-value $=0.035)$ based on a total of 1729 participants, with participant numbers for individual studies ranging from 79 to 1302 participants (Figure 6a). No statistical heterogeneity was found $\left(\mathrm{Chi}^{2}=3.04, d f=3, \mathrm{I}^{2}=1.45 \%, p=0.385\right)$, with the lack of potential publication bias confirmed by Egger's linear regression test (intercept $=-0.18, t=-0.16$, $p=0.887$ ) (Figure 6b). Inversely, in post-menopausal women, no effect was found. ES was 1.10 $((95 \% \mathrm{CI}=0.85-1.43), p$-value $=0.474)$ based on a total of 1486 participants, with participant numbers for individual studies ranging from 70 to 799 participants. No statistical heterogeneity was found $\left(\mathrm{Chi}^{2}=7.166, d f=3, I^{2}=58.09 \%, p=0.067\right)$, with potential publication bias confirmed by Egger's linear regression test (intercept $=2.47, t=2.04, p=0.179$ ).

Table 3. Data extracted from primary studies focusing on menopausal status.

\begin{tabular}{ccccc}
\hline & \multicolumn{2}{c}{ Premenopausal } & \multicolumn{2}{c}{ Postmenopausal } \\
\hline Author, Year & N in Analysis & OR RR HR (CI 95\%) & N in Analysis & OR RR HR (CI 95\%) \\
\hline Li M. et al. 2016 & 267 & OR 0.62 (0.40-0.97) & 405 & OR 1.40 (1.00-1.96) \\
Iwasaki M. et al. 2014 & 79 & OR 1.10 (0.54-2.23) & 212 & OR 1.42 (0.71-2.85) \\
Iwasaki M. et al. 2010 & 81 & HR 0.97 (0.66-1.41) & 70 & HR 1.08 (0.75-1.55) \\
Shrubsole M. et al. 2009 & 1302 & OR 0.87 (0.76-1.00) & 799 & OR 0.88 (0.74-1.04) \\
\hline
\end{tabular}

Abbreviations: $\mathrm{N}$ = number; $\mathrm{OR}$ = odds ratio; $\mathrm{RR}$ = Relative Risk; $\mathrm{HR}$ = Hazard Ratio; $\mathrm{CI}$ = confidence interval

a)

$\begin{array}{rccrcc} & \text { ES } & 95 \% \text { Cl } & \text { W } & \text { Sig. } & \text { N } \\ \text { Iwasaki M. 2010 } & 1.12 & 0.81,1.56 & 33.20 \% & 0.498 & 67,422 \\ \text { Nagano J. 2001 } & 1.00 & 0.67,1.60 & 18.82 \% & 1.000 & 38,540 \\ \text { Suzuki Y.(a) 2004 } & 0.96 & 0.50,1.86 & 8.26 \% & 0.903 & 17,353 \\ \text { Key T.J. 1999 } & 0.86 & 0.62,1.21 & 31.90 \% & 0.377 & 427 \\ \text { Suzuki Y.(b) 2004 } & 0.85 & 0.43,1.66 & 7.82 \% & 0.637 & 24,769 \\ \text { Overall (fixed-effect model) } & 0.97 & 0.81,1.18 & 100.00 \% & 0.783 & 148,511\end{array}$

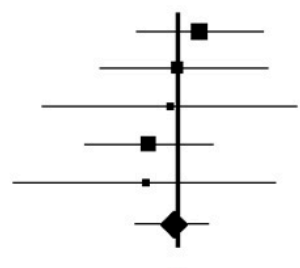

b)

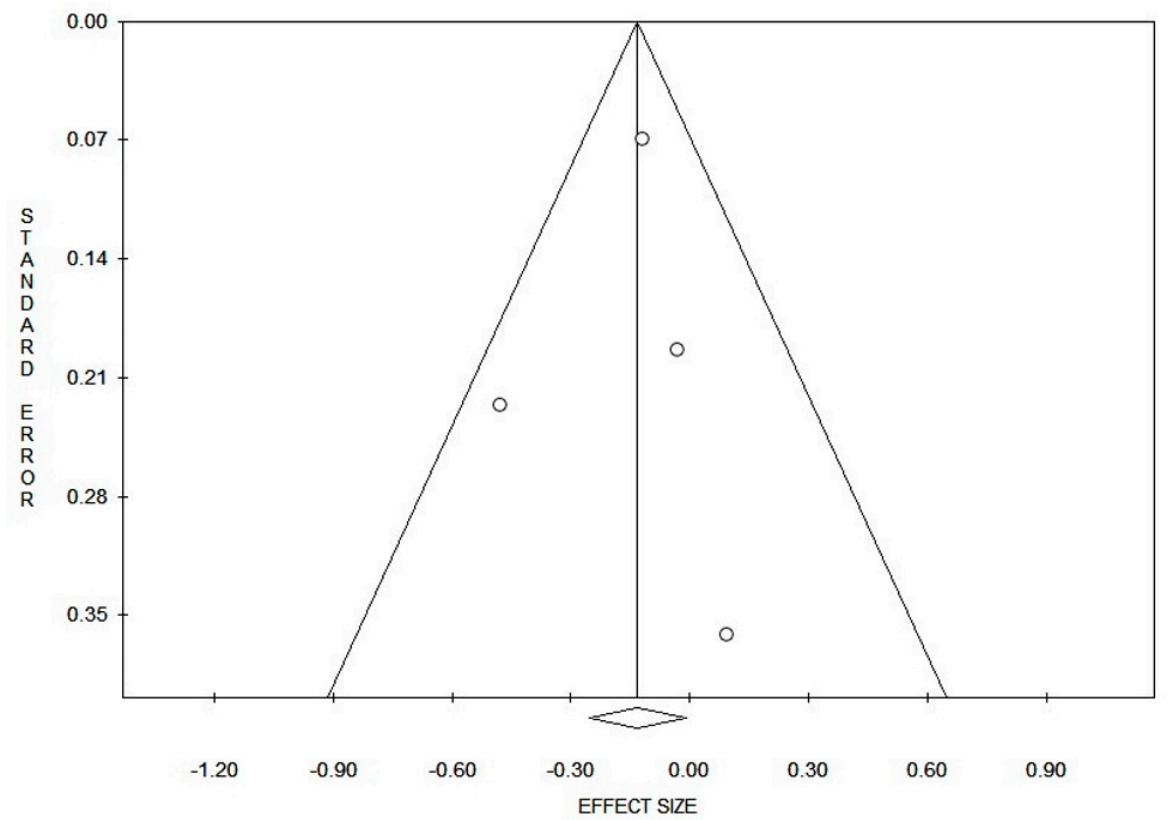

Figure 6. Forest plot (a) of the meta-analysis comparing green tea consumption in breast cancer (BC) (women in pre-menopausal status). Funnel plot (b). Abbreviations: $\mathrm{ES}=$ effect size; $\mathrm{CI}=$ confidence interval; $\mathrm{W}$ = weight; Sig = significance; $\mathrm{N}$ = number. 


\section{Discussion}

The potential beneficial effect of green tea could be related to its high amount of natural phytochemicals, which are preserved because of the absence of fermentation during the production process. Among green tea phytochemicals, polyphenols are the most represented. Several experimental studies have shown that long-term exposure to polyphenols may reduce chronic inflammation and oxidative stress and inhibit the growth, reproduction, and diffusion of cancer cells $[73,74]$. These experimental studies suggest a potentially protective effect of green tea consumption.

This meta-analysis showed the potential effect of green tea consumption in the reduction of breast cancer risk (15\%). Furthermore, because the CI shows the interval within which a true mean is likely to be found, the narrow CIs observed in this systematic review may suggest the precision of ES results. In a sensitivity analysis of case-control studies and those with QS $\geq 5$, a higher protective effect of green tea was found. In particular, a 19\% reduction for case-control studies and a $27 \%$ reduction for studies with QS $\geq 5$ were found.

The majority of cohort studies included in this meta-analysis found a significant reduction of BC recurrence, although data was not confirmed in the case-control studies. The presented results highlight the important role of green tea in tertiary prevention rather than in primary prevention. Actually, green tea appears to reduce $\mathrm{BC}$ incidence in only $3 \%$ of the cases and not in a statistically significant manner. Inversely, the $19 \%$ reduction of BC recurrence appears to be associated with green tea drinking. However, green tea consumption was assessed in all the studies through questionnaire or interview, though only five studies used validated tools. Even though the food frequency questionnaires are cheaper and more manageable instruments to assess dietary intake, they cannot be considered devoid of potential bias. It was not possible to standardize the appropriate consumption value because green tea consumption was recorded in different units in the original studies. Indeed, tea consumption was reported in grams or cups. Furthermore, when cup unit was used as a reference, the amount of tea per cup differed, ranging from $100 \mathrm{~mL}$ to $350 \mathrm{~mL}$. Due to this limitation, it was not possible to perform a dose-response analysis. In the absence of clear evidence answering questions about the right amount and frequency of consumption, it is not possible to estimate the amount of tea that may provide preventive effects. Indeed, a comparison between the highest intake of green tea, as reported in the original papers, with no consumption or lowest intake was performed. Nevertheless, a subgroup analysis that only included studies with green tea intake higher than 5 cups/day was carried out. Moreover, in order to minimize potential bias, adjusted data were collected from the original studies. In particular, attention was focused on adjustment for age, Body Mass Index (BMI), parity, history of BC, and smoking habit. Nonetheless, several studies also adjusted for other potential confounding factors such as age of menarche, age of first pregnancy, number of births, and menopausal status.

This meta-analysis shows similar results previously obtained in another meta-analysis performed in 2010 [75]. However, in the study conducted by Ogunleye et al., only one database was consulted and a limited number of articles were retrieved. In addition, several subgroup analyses were performed.

\section{Strengths and Limitations of the Study}

Some limitations have to be taken into account when reading this meta-analysis. First, the majority of the studies were conducted in Asia, mostly China and Japan, which represent the largest green tea production and consumption areas. Only one study was conducted in the USA, and no studies were conducted in Europe. This highlights the importance of performing similar studies in other ethnic populations in order to underline any potential differences. This is particularly true in determining whether other antioxidants consumed in the diet can play a confounding role. Another limitation is that self-reported green tea intake by FFQ was measured at baseline only, and the duration of tea consumption is not known. This can result in errors during both reporting and classification. Moreover, the different amounts of green tea consumed as higher intake in the original studies could have been defined in post hoc analysis. 
This is likely the first meta-analysis also assessing green tea intake, the risk of BC in post-menopausal women, and $\mathrm{BC}$ stage. Another significant strength of the present study is that the risk for $\mathrm{BC}$ and recurrence were considered both together and separately. However, due to the absence of information in the original studies, it was not possible to evaluate the effect of green tea based on $\mathrm{BC}$ stage at diagnosis.

All the above-mentioned limitations can explain the different results between studies and the absence of statistical significance when looking at the different states of menopause or the preferable amount of intake. Future studies need to focus on these particular aspects. Moreover, studies with higher numbers of participants need to be performed.

This meta-analysis has some important strengths, such as the large number of participants $(163,810)$, with participants ranging from 20-87 years of age. Another strength is represented by the sensitivity analysis that was conducted in population subgroups. Furthermore, results in population subgroups are important for public health decision-makers to help them implement effective evidence-based strategies for selected population groups (e.g., high-risk participants), thereby enhancing the quality and efficiency of interventions [76].

According to the present results, the highest protective effect of green tea was found in BC recurrence prevention. This is in line with previous studies, where the authors demonstrated an anti-neoplastic effect of green tea through a reduction in viability and proliferation of BC cells [77-79]. In particular, a previous review on epigenetic modification by epigallocatechin on $\mathrm{BC}$ cell lines revealed a significantly greater inhibition of $B C$ cell growth. Moreover, the inhibitory effect of epigallocatechin on DNA methyltransferase in BC cells is supposed to suppress proliferation through apoptosis induction as well [20]. Indeed, a meta-analysis evaluating the role of epigallocatechin in apoptosis induction in BC cell lines found a positive significant association with a ratio of mean (RoM) of 2.84 (95\% CI 2.60-3.10) for BC cells treated with $20 \mu \mathrm{M}$ of epigallocatechin [21].

Further epidemiological studies are needed to confirm these results and to quantify the impact of behavior change in this disease. Moreover, the majority of the included studies performed a satisfactory follow-up (a mean of approximately 10 years).

\section{Conclusion}

A statistically significant inverse association between green tea and BC was found. This finding is in line with previous publications. However, the results are not always concordant, especially when looking at cohort studies or selecting only those studies analyzing the diagnosis of $\mathrm{BC}$ recurrence. However, a beneficial effect of green tea cannot be excluded, in particular because when only case-control studies were analyzed, a statistically significant protective effect was observed. Indeed, the importance of case-control studies in defining the causal relationship between exposure and event is well known. Therefore, further studies are required to confirm these data before any public recommendations can be made. In particular, it is important to conduct studies with greater numbers of people, in different countries, and with a serial evaluation of lifetime green tea consumption.

Moreover, as tea is one of the most popular beverages in the world and may possess many chemo-preventive qualities, a better understanding of the mechanisms might improve the utilization of green tea in breast cancer primary and recurrence prevention. At the clinical level, results from this systematic review and meta-analysis may be useful to design clinical trials aimed at ascertaining the potential role of green tea as an adjuvant in breast cancer therapy and pave the way to novel preventive strategies for breast cancer.

Supplementary Materials: The following are available online at http://www.mdpi.com/2072-6643/10/12/1886/s1. Table S1: Studies excluded with reason.

Author Contributions: Conceptualization, V.G., D.N., A.A.; Methodology, V.G.; Formal Analysis, V.G. and D.N.; investigation, V.G., A.A., M.A.; Writing Original Draft preparation, V.G. and D.N.; Writing-Review \& Editing, M.V., M.M., R.S.; Visualization, V.G.; Supervision, S.R. All authors approved the final version of the manuscript.

Funding: This research received no external funding. 
Acknowledgments: The authors are grateful to Christina Drace, Veneto Institute of Oncology IOV-IRCCS, Padua, Italy for the English revision and to Valeria Parisi, University of Milan, for support with the figures.

Conflicts of Interest: The authors declare no conflict of interest.

\section{References}

1. Namita, P.; Mukesh, R.; Vijay, K.J. Camellia Sinensis (Green Tea): A Review. Glob. J. Pharmacol. 2012, 6, 52-59.

2. Saeed, M.; Naveed, M.; Arif, M.; Kakar, M.U.; Manzoor, R.; Abd El-Hack, M.E.; Alagawany, M.; Tiwari, R.; Khandia, R.; Munjal, A.; et al. Green tea (Camellia sinensis) and 1-theanine: Medicinal values and beneficial applications in humans-A comprehensive review. Biomed. Pharmacother. 2017, 95, 1260-1275. [CrossRef] [PubMed]

3. Sun, C.L.; Yuan, J.M.; Koh, W.P.; Yu, M.C. Green tea, black tea and breast cancer risk: A meta-analysis of epidemiological studies. Carcinogenesis 2006, 27, 1310-1315. [CrossRef]

4. Bhattacharyyaa, N.; Sethb, S.; Tudub, B.; Tamulyc, P.; Janaa, A.; Ghosha, D.; Bandyopadhyayb, R.; Bhuyand, M. Monitoring of black tea fermentation process using electronic nose. J. Food Eng. 2007, 80, 1146-1156. [CrossRef]

5. Juneja, L.R.; Kapoor, M.P.; Okubo, T.; Rao, T. Green Tea Polyphenols: Nutraceuticals of Modern Life; CRC Press: Boca Raton, FL, USA, 2016.

6. Rätsch, C. The Encyclopedia of Psychoactive Plants: Ethnopharmacology and Its Applications; Park Street Press: Rochester, VT, USA, 2004.

7. Weisburger, J.H.; Chung, F.L. Mechanisms of chronic disease causation by nutritional factors and tobacco products and their prevention by tea polyphenols. Food Chem. Toxicol. 2002, 40, 1145-1154. [CrossRef]

8. Food and Agriculture Organization of the United Nations (FAO). World Tea Production and Trade. Current and Future Development; Food and Agriculture Organization of the United Nations (FAO): Rome, Italy, 2015.

9. Sumpio, B.E.; Cordova, A.C.; Berke-Schlessel, D.W.; Qin, F.; Chen, Q.H. Green tea, the "Asian paradox," and cardiovascular disease. J. Am. Coll. Surg. 2006, 202, 813-825. [CrossRef] [PubMed]

10. Dufresne, C.J.; Farnworth, E.R. A review of latest research findings on the health promotion properties of tea. J. Nutr. Biochem. 2001, 12, 404-421. [CrossRef]

11. Cabrera, C.; Artacho, R.; Gimenez, R. Beneficial effects of green tea-A review. J. Am. Coll. Nutr. 2006, 25, 79-99. [CrossRef]

12. Yuan, J.M.; Sun, C.; Butler, L.M. Tea and cancer prevention: Epidemiological studies. Pharmacol. Res. 2011, 64, 123-135. [CrossRef]

13. Yang, C.S.; Wang, H.; Li, G.X.; Yang, Z.; Guan, F.; Jin, H. Cancer prevention by tea: Evidence from laboratory studies. Pharmacol. Res. 2011, 64, 113-122. [CrossRef]

14. Fon Sing, M.; Yang, W.S.; Gao, S.; Gao, J.; Xiang, Y.B. Epidemiological studies of the association between tea drinking and primary liver cancer: A meta-analysis. Eur. J. Cancer Prev. 2011, 20, 157-165. [CrossRef]

15. Mann, C.D.; Neal, C.P.; Garcea, G.; Manson, M.M.; Dennison, A.R.; Berry, D.P. Phytochemicals as potential chemopreventive and chemotherapeutic agents in hepatocarcinogenesis. Eur. J. Cancer Prev. 2009, 18, $13-25$. [CrossRef]

16. Yiannakopoulou, E.C. Interaction of green tea catechins with breast cancer endocrine treatment: A systematic review. Pharmacology 2014, 94, 245-248. [CrossRef] [PubMed]

17. Jacob, S.A.; Khan, T.M.; Lee, L.H. The Effect of Green Tea Consumption on Prostate Cancer Risk and Progression: A Systematic Review. Nutr. Cancer 2017, 69, 353-364. [CrossRef]

18. Fujiki, H.; Watanabe, T.; Sueoka, E.; Rawangkan, A.; Suganuma, M. Cancer Prevention with Green Tea and Its Principal Constituent, EGCG: From Early Investigations to Current Focus on Human Cancer Stem Cells. Mol. Cells 2018, 41, 73-82. [CrossRef]

19. Sur, S.; Panda, C.K. Molecular aspects of cancer chemopreventive and therapeutic efficacies of tea and tea polyphenols. Nutrition 2017, 43-44, 8-15. [CrossRef] [PubMed]

20. Gianfredi, V.; Nucci, D.; Vannini, S.; Villarini, M.; Moretti, M. In vitro Biological Effects of Sulforaphane (SFN), Epigallocatechin-3-gallate (EGCG), and Curcumin on Breast Cancer Cells: A Systematic Review of the Literature. Nutr. Cancer 2017, 69, 969-978. [CrossRef] 
21. Gianfredi, V.; Vannini, S.; Moretti, M.; Villarini, M.; Bragazzi, N.L.; Izzotti, A.; Nucci, D. Sulforaphane and Epigallocatechin Gallate Restore Estrogen Receptor Expression by Modulating Epigenetic Events in the Breast Cancer Cell Line MDA-MB-231: A Systematic Review and Meta-Analysis. J. Nutrigenet. Nutrigenom. 2017, 10, 126-135. [CrossRef]

22. Chikara, S.; Nagaprashantha, L.D.; Singhal, J.; Horne, D.; Awasthi, S.; Singhal, S.S. Oxidative stress and dietary phytochemicals: Role in cancer chemoprevention and treatment. Cancer Lett. 2018, 413, 122-134. [CrossRef]

23. Rashidi, B.; Malekzadeh, M.; Goodarzi, M.; Masoudifar, A.; Mirzaei, H. Green tea and its anti-angiogenesis effects. Biomed. Pharmacother. 2017, 89, 949-956. [CrossRef]

24. Bray, F.; Ferlay, J.; Soerjomataram, I.; Siegel, R.L.; Torre, L.A.; Jemal, A. Global cancer statistics 2018: GLOBOCAN estimates of incidence and mortality worldwide for 36 cancers in 185 countries. Cancer J. Clin. 2018, 68, 394-424. [CrossRef]

25. Winters, S.; Martin, C.; Murphy, D.; Shokar, N.K. Breast Cancer Epidemiology, Prevention, and Screening. Prog. Mo. Biol. Transl. Sci. 2017, 151, 1-32. [CrossRef]

26. Shamseer, L.; Moher, D.; Clarke, M.; Ghersi, D.; Liberati, A.; Petticrew, M.; Shekelle, P.; Stewart, L.A.; Group, P.-P. Preferred reporting items for systematic review and meta-analysis protocols (PRISMA-P) 2015: Elaboration and explanation. BMJ 2015, 350, g7647. [CrossRef]

27. Stroup, D.F.; Berlin, J.A.; Morton, S.C.; Olkin, I.; Williamson, G.D.; Rennie, D.; Moher, D.; Becker, B.J.; Sipe, T.A.; Thacker, S.B. Meta-analysis of observational studies in epidemiology: A proposal for reporting. Meta-analysis Of Observational Studies in Epidemiology (MOOSE) group. JAMA 2000, 283, 2008-2012. [CrossRef]

28. Brown, P.; Brunnhuber, K.; Chalkidou, K.; Chalmers, I.; Clarke, M.; Fenton, M.; Forbes, C.; Glanville, J.; Hicks, N.J.; Moody, J.; et al. How to formulate research recommendations. BMJ 2006, 333, 804-806. [CrossRef]

29. Wong, W.C.; Cheung, C.S.; Hart, G.J. Development of a quality assessment tool for systematic reviews of observational studies (QATSO) of HIV prevalence in men having sex with men and associated risk behaviours. Themes Epidemiol. 2008, 5, 23. [CrossRef]

30. von Elm, E.; Altman, D.G.; Egger, M.; Pocock, S.J.; Gotzsche, P.C.; Vandenbroucke, J.P.; Initiative, S. The Strengthening the Reporting of Observational Studies in Epidemiology (STROBE) Statement: Guidelines for reporting observational studies. Int. J. Surg. 2014, 12, 1495-1499. [CrossRef]

31. Buitrago-Lopez, A.; Sanderson, J.; Johnson, L.; Warnakula, S.; Wood, A.; Di Angelantonio, E.; Franco, O.H. Chocolate consumption and cardiometabolic disorders: Systematic review and meta-analysis. BMJ 2011, 343, d4488. [CrossRef]

32. Higgins, J.P.; Thompson, S.G.; Deeks, J.J.; Altman, D.G. Measuring inconsistency in meta-analyses. BMJ 2003, 327, 557-560. [CrossRef]

33. Arthur, R.; Kirsh, V.A.; Rohan, T.E. Associations of coffee, tea and caffeine intake with risk of breast, endometrial and ovarian cancer among Canadian women. Cancer Epidemiol. 2018, 56, 75-82. [CrossRef]

34. Ronco, A.L.; Stefani, E.D.; Mendoza, B.; Vazquez, A.; Abbona, E.; Sanchez, G.; Rosa, A.D. Mate and Tea Intake, Dietary Antioxidants and Risk of Breast Cancer: A Case-Control Study. Asian Pac. J. Cancer Prev. 2016, 17, 2923-2933. [CrossRef]

35. Bhoo-Pathy, N.; Peeters, P.H.; Uiterwaal, C.S.; Bueno-de-Mesquita, H.B.; Bulgiba, A.M.; Bech, B.H.; Overvad, K.; Tjonneland, A.; Olsen, A.; Clavel-Chapelon, F.; et al. Coffee and tea consumption and risk of pre- and postmenopausal breast cancer in the European Prospective Investigation into Cancer and Nutrition (EPIC) cohort study. Breast Cancer Res. 2015, 17, 15. [CrossRef]

36. Oh, J.K.; Sandin, S.; Strom, P.; Lof, M.; Adami, H.O.; Weiderpass, E. Prospective study of breast cancer in relation to coffee, tea and caffeine in Sweden. Int. J. Cancer 2015, 137, 1979-1989. [CrossRef]

37. Touvier, M.; Druesne-Pecollo, N.; Kesse-Guyot, E.; Andreeva, V.A.; Fezeu, L.; Galan, P.; Hercberg, S.; Latino-Martel, P. Dual association between polyphenol intake and breast cancer risk according to alcohol consumption level: A prospective cohort study. Breast Cancer Res. Treat. 2013, 137, 225-236. [CrossRef]

38. Fagherazzi, G.; Touillaud, M.S.; Boutron-Ruault, M.C.; Clavel-Chapelon, F.; Romieu, I. No association between coffee, tea or caffeine consumption and breast cancer risk in a prospective cohort study. Public Heal. Nutr. 2011, 14, 1315-1320. [CrossRef] 
39. Boggs, D.A.; Palmer, J.R.; Stampfer, M.J.; Spiegelman, D.; Adams-Campbell, L.L.; Rosenberg, L. Tea and coffee intake in relation to risk of breast cancer in the Black Women's Health Study. Cancer Causes Control 2010, 21, 1941-1948. [CrossRef]

40. Luo, J.; Gao, Y.T.; Chow, W.H.; Shu, X.O.; Li, H.; Yang, G.; Cai, Q.; Rothman, N.; Cai, H.; Shrubsole, M.J.; et al. Urinary polyphenols and breast cancer risk: Results from the Shanghai Women's Health Study. Breast Cancer Res. Treat. 2010, 120, 693-702. [CrossRef]

41. Luo, J.F.; Gao, Y.T.; Chow, W.H.; Shu, X.O.; Li, H.L.; Yang, G.; Cai, Q.Y.; Li, G.L.; Rothman, N.; Cai, H.; et al. Urinary polyphenols, glutathione S-transferases copy number variation, and breast cancer risk: Results from the Shanghai women's health study. Mol. Carcinog. 2012, 51, 379-388. [CrossRef]

42. Pathy, N.B.; Peeters, P.; van Gils, C.; Beulens, J.W.J.; van der Graaf, Y.; Bueno-de-Mesquita, B.; Bulgiba, A.; Uiterwaal, C.S.P.M. ; D., V.G.Y.; Bueno-de-Mesquita, H.B.; Bulgiba, A.; uiterwaal, Cofee and tea intake and risk of breast cancer. Breast Cancer Res. Treat. 2010, 121, 461-467. [CrossRef]

43. Michels, K.B.; Holmberg, L.; Bergkvist, L.; Wolk, A. Coffee, tea, and caffeine consumption and breast cancer incidence in a cohort of Swedish women. Ann. Epidemiol. 2002, 12, 21-26. [CrossRef]

44. Kumar, N.; Titus-Ernstoff, L.; Newcomb, P.A.; Trentham-Dietz, A.; Anic, G.; Egan, K.M. Tea consumption and risk of breast cancer. Cancer Epidemiol. Biomarkers Prev. 2009, 18, 341-345. [CrossRef]

45. Ganmaa, D.; Willett, W.C.; Li, T.Y.; Feskanich, D.; van Dam, R.M.; Lopez-Garcia, E.; Hunter, D.J.; Holmes, M.D. Coffee, tea, caffeine and risk of breast cancer: A 22-year follow-up. Int. J. Cancer 2008, 122, 2071-2076. [CrossRef]

46. Zheng, W.; Doyle, T.J.; Kushi, L.H.; Sellers, T.A.; Hong, C.P.; Folsom, A.R. Tea consumption and cancer incidence in a prospective cohort study of postmenopausal women. Am. J. Epidemiol. 1996, 144, 175-182. [CrossRef]

47. McLaughlin, C.C.; Mahoney, M.C.; Nasca, P.C.; Metzger, B.B.; Baptiste, M.S.; Field, N.A. Breast cancer and methylxanthine consumption. Cancer Causes Control 1992, 3, 175-178. [CrossRef]

48. Wang, Y.; Gapstur, S.M.; Gaudet, M.M.; Peterson, J.J.; Dwyer, J.T.; McCullough, M.L. Evidence for an Association of Dietary Flavonoid Intake with Breast Cancer Risk by Estrogen Receptor Status Is Limited. J. Nutr. 2014, 144, 1603-1611. [CrossRef]

49. Crew, K.D.; Brown, P.; Greenlee, H.; Bevers, T.B.; Arun, B.; Hudis, C.; McArthur, H.L.; Chang, J.; Rimawi, M.; Vornik, L.; et al. Phase IB Randomized, Double-Blinded, Placebo-Controlled, Dose Escalation Study of Polyphenon E in Women with Hormone Receptor-Negative Breast Cancer. Cancer Prev. Res. 2012, 5, 1144-1154. [CrossRef]

50. Crew, K.D.; Ho, K.A.; Brown, P.; Greenlee, H.; Bevers, T.B.; Arun, B.; Sneige, N.; Hudis, C.; McArthur, H.L.; Chang, J.; et al. Effects of a green tea extract, Polyphenon E, on systemic biomarkers of growth factor signalling in women with hormone receptor-negative breast cancer. J. Hum. Nutr. Diet. 2015, 28, 272-282. [CrossRef]

51. Lazzeroni, M.; Guerrieri-Gonzaga, A.; Gandini, S.; Johansson, H.; Serrano, D.; Cazzaniga, M.; Aristarco, V.; Macis, D.; Mora, S.; Caldarella, P.; et al. A Presurgical Study of Lecithin Formulation of Green Tea Extract in Women with Early Breast Cancer. Cancer Prev. Res. 2017, 10, 363-369. [CrossRef]

52. Samavat, H.; Dostal, A.M.; Wang, R.W.; Bedell, S.; Emory, T.H.; Ursin, G.; Torkelson, C.J.; Gross, M.D.; Le, C.T.; Yu, M.C.; et al. The Minnesota Green Tea Trial (MGTT), a randomized controlled trial of the efficacy of green tea extract on biomarkers of breast cancer risk: Study rationale, design, methods, and participant characteristics. Cancer Causes Control 2015, 26, 1405-1419. [CrossRef]

53. Samavat, H.; Ursin, G.; Emory, T.H.; Lee, E.; Wang, R.W.; Torkelson, C.J.; Dostal, A.M.; Swenson, K.; Le, C.T.; Yang, C.S.; et al. A Randomized Controlled Trial of Green Tea Extract Supplementation and Mammographic Density in Postmenopausal Women at Increased Risk of Breast Cancer. Cancer Prev. Res. 2017, 10, 710-718. [CrossRef]

54. Chen, X.; Lu, W.; Zheng, Y.; Gu, K.; Chen, Z.; Zheng, W.; Shu, X.O. Exercise, tea consumption, and depression among breast cancer survivors. J. Clin. Oncol. 2010, 28, 991-998. [CrossRef]

55. Stendell-Hollis, N.R.; Thomson, C.A.; Thompson, P.A.; Bea, J.W.; Cussler, E.C.; Hakim, I.A. Green tea improves metabolic biomarkers, not weight or body composition: A pilot study in overweight breast cancer survivors. J. Hum. Nutr. Diet. 2010, 23, 590-600. [CrossRef] 
56. Zhu, W.Q.; Jia, L.; Chen, G.X.; Zhao, H.X.; Sun, X.R.; Meng, X.J.; Zhao, X.G.; Xing, L.G.; Yu, J.M.; Zheng, M.Z. Epigallocatechin-3-gallate ameliorates radiation-induced acute skin damage in breast cancer patients undergoing adjuvant radiotherapy. Oncotarget 2016, 7, 48607-48613. [CrossRef]

57. Mayo Clinic. Drinking coffee and tea doesn't raise breast cancer risk. Mayo Clinic Women's Healthsource 2008, 12,3 .

58. Iwasaki, M.; Inoue, M.; Sasazuki, S.; Miura, T.; Sawada, N.; Yamaji, T.; Shimazu, T.; Willett, W.C.; Tsugane, S. Plasma tea polyphenol levels and subsequent risk of breast cancer among Japanese women: A nested case-control study. Breast Cancer Res. Treat. 2010, 124, 827-834. [CrossRef]

59. Zhang, M.; Huang, J.; Xie, X.; Holman, C.D.J. Dietary intakes of mushrooms and green tea combine to reduce the risk of breast cancer in Chinese women. Int. J. Cancer 2009, 124, 1404-1408. [CrossRef]

60. Suzuki, Y.; Tsubono, Y.; Nakaya, N.; Suzuki, Y.; Koizumi, Y.; Tsuji, I. Green tea and the risk of breast cancer: Pooled analysis of two prospective studies in Japan. Br. J. Cancer 2004, 90, 1361-1363. [CrossRef]

61. Inoue, M.; Tajima, K.; Mizutani, M.; Iwata, H.; Iwase, T.; Miura, S.; Hirose, K.; Hamajima, N.; Tominaga, S. Regular consumption of green tea and the risk of breast cancer recurrence: Follow-up study from the Hospital-based Epidemiologic Research Program at Aichi Cancer Center (HERPACC), Japan. Cancer Lett. 2001, 167, 175-182. [CrossRef]

62. Iwasaki, M.; Inoue, M.; Sasazuki, S.; Sawada, N.; Yamaji, T.; Shimazu, T.; Willett, W.C.; Tsugane, S. Green tea drinking and subsequent risk of breast cancer in a population-based cohort of Japanese women. Breast Cancer Res. 2010, 12, R88. [CrossRef]

63. Iwasaki, M.; Mizusawa, J.; Kasuga, Y.; Yokoyama, S.; Onuma, H.; Nishimura, H.; Kusama, R.; Tsugane, S. Green tea consumption and breast cancer risk in Japanese women: A case-control study. Nutr. Cancer 2014, 66, 57-67. [CrossRef]

64. Key, T.J.; Sharp, G.B.; Appleby, P.N.; Beral, V.; Goodman, M.T.; Soda, M.; Mabuchi, K. Soya foods and breast cancer risk: A prospective study in Hiroshima and Nagasaki, Japan. Br. J. Cancer 1999, 81, 1248-1256. [CrossRef]

65. Nagano, J.; Kono, S.; Preston, D.L.; Mabuchi, K. A prospective study of green tea consumption and cancer incidence, Hiroshima and Nagasaki (Japan). Cancer Causes Control 2001, 12, 501-508. [CrossRef]

66. Nakachi, K.; Suemasu, K.; Suga, K.; Takeo, T.; Imai, K.; Higashi, Y. Influence of drinking green tea on breast cancer malignancy among Japanese patients. Jpn. J. Cancer Res. 1998, 89, 254-261. [CrossRef]

67. Inoue, M.; Robien, K.; Wang, R.; Van Den Berg, D.J.; Koh, W.P.; Yu, M.C. Green tea intake, MTHFR/TYMS genotype and breast cancer risk: The Singapore Chinese Health Study. Carcinogenesis 2008, 29, 1967-1972. [CrossRef]

68. Li, M.; Tse, L.A.; Chan, W.C.; Kwok, C.H.; Leung, S.L.; Wu, C.; Yu, W.C.; Yu, I.T.; Yu, C.H.; Wang, F.; et al. Evaluation of breast cancer risk associated with tea consumption by menopausal and estrogen receptor status among Chinese women in Hong Kong. Cancer Epidemiol. 2016, 40, 73-78. [CrossRef]

69. Shrubsole, M.J.; Lu, W.; Chen, Z.; Shu, X.O.; Zheng, Y.; Dai, Q.; Cai, Q.; Gu, K.; Ruan, Z.X.; Gao, Y.T.; et al. Drinking green tea modestly reduces breast cancer risk. J. Nutr. 2009, 139, 310-316. [CrossRef]

70. Yuan, J.M.; Koh, W.P.; Sun, C.L.; Lee, H.P.; Yu, M.C. Green tea intake, ACE gene polymorphism and breast cancer risk among Chinese women in Singapore. Carcinogenesis 2005, 26, 1389-1394. [CrossRef]

71. Zhang, M.; Holman, C.D.; Huang, J.P.; Xie, X. Green tea and the prevention of breast cancer: A case-control study in Southeast China. Carcinogenesis 2007, 28, 1074-1078. [CrossRef]

72. Wu, A.H.; Yu, M.C.; Tseng, C.C.; Hankin, J.; Pike, M.C. Green tea and risk of breast cancer in Asian Americans. Int. J. Cancer 2003, 106, 574-579. [CrossRef]

73. Mao, X.; Gu, C.; Chen, D.; Yu, B.; He, J. Oxidative stress-induced diseases and tea polyphenols. Oncotarget 2017, 8, 81649-81661. [CrossRef]

74. Ma, Y.; Zhao, L.; Gao, M.; Loor, J.J. Tea polyphenols protect bovine mammary epithelial cells from hydrogen peroxide-induced oxidative damage in vitro. J. Anim. Sci. 2018, 96, 4159-4172. [CrossRef]

75. Ogunleye, A.A.; Xue, F.; Michels, K.B. Green tea consumption and breast cancer risk or recurrence: A meta-analysis. Breast Cancer Res. Treat. 2010, 119, 477-484. [CrossRef]

76. Parks, R.G.; Tabak, R.G.; Allen, P.; Baker, E.A.; Stamatakis, K.A.; Poehler, A.R.; Yan, Y.; Chin, M.H.; Harris, J.K.; Dobbins, M.; et al. Enhancing evidence-based diabetes and chronic disease control among local health departments: A multi-phase dissemination study with a stepped-wedge cluster randomized trial component. Implement. Sci. 2017, 12, 122. [CrossRef] 
77. Horakova, D.; Bouchalova, K.; Cwiertka, K.; Stepanek, L.; Vlckova, J.; Kollarova, H. Risks and protective factors for triple negative breast cancer with a focus on micronutrients and infections. Biomed. Pap. Med. Fac. Univ. Palacky Olomouc Czech Repub. 2018, 162, 83-89. [CrossRef]

78. Schroder, L.; Marahrens, P.; Koch, J.G.; Heidegger, H.; Vilsmeier, T.; Phan-Brehm, T.; Hofmann, S.; Mahner, S.; Jeschke, U.; Richter, D.U. Effects of green tea, matcha tea and their components epigallocatechin gallate and quercetin on MCF7 and MDA-MB-231 breast carcinoma cells. Oncol. Rep. 2018. [CrossRef]

79. Wei, R.; Mao, L.; Xu, P.; Zheng, X.; Hackman, R.M.; Mackenzie, G.G.; Wang, Y. Suppressing glucose metabolism with epigallocatechin-3-gallate (EGCG) reduces breast cancer cell growth in preclinical models. Food Funct. 2018, 9, 5682-5696. [CrossRef]

(C) 2018 by the authors. Licensee MDPI, Basel, Switzerland. This article is an open access article distributed under the terms and conditions of the Creative Commons Attribution (CC BY) license (http:/ / creativecommons.org/licenses/by/4.0/). 